\title{
SÍNTESIS DE MEMBRANA DE LÍQUIDOS IÓNICOS POLIMERIZADOS (PILM) CON EL INDICADOR AMARILLO BRILLANTE PARA DETECCIÓN DE pH
}

Andrea G. Yipmantín Ojeda ${ }^{a^{*}}$, Enrique Plaza Villanueva ${ }^{\mathrm{b}}$, Víctor R. García Villegas ${ }^{\mathrm{c}}$, Holger J. Maldonado García ${ }^{\mathrm{a}, \mathrm{c}}$.

\section{RESUMEN}

Se ha desarrollado una membrana de líquidos iónicos polimerizados (PILM) a partir de la copolimerización del bromuro de 3-butil-1-vinilimidazolio y acrilonitrilo, posteriormente cargada con el indicador amarillo brillante $(\mathrm{AB})$ mediante un proceso de intercambio iónico (PILM-AB). El objetivo de este proceso fue la utilización de la membrana en la medición de $\mathrm{pH}$ en soluciones acuosas en el rango 6,5 a 8,0; cercano a condiciones neutras. Las membranas crudas PILM y cargadas con el indicador PILM-AB fueron caracterizadas mediante FT-IR y la respuesta de las membranas cargadas PILM-AB respecto al cambio de $\mathrm{pH}$ fue medida utilizando un Espectrofotómetro UV-Vis.

Palabras clave: Membrana, $\mathrm{pH}$, indicador, líquidos iónicos polimerizados, PILM.

\section{SYNTHESIS OF A POLYMERIZED IONIC LIQUID MEMBRANE (PILM) WITH BRILLIANT YELLOW FOR ITS USE AS A pH INDICATOR}

\begin{abstract}
A polymerized ionic liquid membrane (PILM) has been synthesized by copolymerization of 3-butyl-1-vinylimidazolium bromide with acrylonitrile and then loaded with brilliant yellow dye $(\mathrm{AB})$ describing an anion exchange process. The main objetive of this synthesis is the measurement of $\mathrm{pH}$ in aqueous solutions between $\mathrm{pH}$ 6,5 and 8,0; close to neutral conditions. Both PILM and PILM-AB membranes were characterized by FT-IR spectroscopy and their pH-response was measured by UV-Vis spectrophotometry.
\end{abstract}

Key words: Membrane, $\mathrm{pH}$, dyes, polymerized ionic liquids, PILM.

\section{INTRODUCCIÓN}

En los últimos 20 años, se ha investigado en profundidad las características, propiedades y aplicaciones de los líquidos iónicos (ionic liquid - IL). Estos se definen como sales con punto de fusión menor a $100^{\circ} \mathrm{C}$, alta conductividad iónica, estabilidad térmica, no volátil, no

\footnotetext{
a Facultad de Ciencias y Filosofía “Alberto Cazorla Talleri”, Universidad Peruana Cayetano Heredia, Lima 31. andm2k@gmail.com

${ }^{\mathrm{b}}$ Molident Odontología Integral S.A.C., Lima 12.

c Facultad de Química e Ingeniería Química, Universidad Nacional Mayor de San Marcos, Lima 1.
} 
inflamable y con alta polarizabilidad. ${ }^{1}$ Las propiedades de los líquidos iónicos dependen de su composición, presentando comúnmente cationes como imidazolio, piridinio, alquilamonio, alquilfosfonio, pirrolidinio y guanidinio. Los aniones correspondientes incluyen haluros, poliatómicos inorgánicos, aniones de ácidos minerales y aniones orgánicos, los cuales pueden ser modificados mediante procesos de intercambio iónico y purificación, de acuerdo a las necesidades específicas de diseño. ${ }^{2}$ Las principales aplicaciones de los líquidos iónicos se presentan en las áreas de electroquímica, ${ }^{3}$ catálisis, ${ }^{4}$ biotecnología ${ }^{5}$ e industria farmacéutica, ${ }^{6}$ así como en el tratamiento de aguas debido a su baja toxicidad. ${ }^{7}$

En la década de 1970, se inició la investigación acerca de la polimerización de monómeros catiónicos de vinilo ${ }^{2}$ y no fue sino hasta 1998 , cuando $\mathrm{Ohno}^{8}$ reportó la modificación de líquidos iónicos mediante su polimerización para formar películas de conductividad alta (polymerized ionic liquids - PILs), que la publicación de investigaciones acerca de estos compuestos, estrategias de síntesis y aplicaciones se ha desarrollado significativamente., $, 10,11$

Las principales estrategias de síntesis de los PILs son la modificación química de polímeros existentes y la polimerización directa de monómeros de IL. ${ }^{2}$ EL primer enfoque tiene como ventaja la posibilidad de controlar las características del polímero antes de la modificación para generar los PILs, mientras que la polimerización directa permite utilizar directamente líquidos iónicos con unidades polimerizables para obtener diferentes productos como homopolímeros, copolímeros o terpolímeros de PILs.

La estrategia de polimerización directa ha sido utilizada para obtener homopolímeros de sales de vinilimidazolio ${ }^{12}$ y aplicada en la síntesis de membranas de líquidos iónicos polimerizados (PILM) conteniendo indicadores de $\mathrm{pH}$ como rojo de cresol, anaranjado de metilo y azul de bromotimol, para su utilización en el rango de $\mathrm{pH}$ de 1 a 14 , en incrementos unitarios de $\mathrm{pH} .{ }^{13}$

El objetivo del presente artículo fue optimizar los parámetros relativos a la síntesis de las membranas PILM mediante copolimerización directa de una sal de vinilimidazolio y acrilonitrilo, cargadas con el indicador amarillo brillante, para su evaluación como un indicador soportado reversible en soluciones acuosas en el rango directo de $\mathrm{pH}$ de 6,5 a 8,0 e inverso de $\mathrm{pH} 8,0$ a 6,5; cercano a condiciones neutrales, similares al pH fisiológico.

\section{PARTE EXPERIMENTAL}

\section{Síntesis de la membrana de líquidos iónicos polimerizados (PILM)}

Se preparó el bromuro de 3-butil-1-vinilimidazolio mezclando cantidades equivalentes de 1-vinilimidazol y 1-bromobutano (Sigma Aldrich) en un baño de hielo por 1 hora; luego a temperatura ambiente por 24 horas. ${ }^{12}$ El producto de esta reacción fue mezclado con acrilonitrilo (1:1), 1-4\% (m/m) de divinilbenceno (Sigma Aldrich) y 0,5\% de isobutil éter de benzoína (TCI), colocado en un molde de vidrio con cintas espaciadoras de $75 \mu \mathrm{m}$ y luego irradiado con luz UV $(254 \mathrm{~nm})$ por 10 minutos. $^{13}$ 


\section{Proceso de intercambio iónico}

La membrana resultante se colocó en una solución etanólica de Amarillo Brillante (AB) (Sigma Aldrich) saturada con $\mathrm{N}_{2}$ por 5 minutos y luego se lavó con etanol para remover el exceso de indicador de la superficie de la membrana (PILM-AB).

\section{Caracterización de PILM y PILM-AB}

Las membranas PILM y PILM-AB fueron caracterizadas mediante Espectroscopía de Infrarrojo FT-IR, empleando discos de $\mathrm{KBr}$.

\section{Pruebas de respuesta al pH en soluciones acuosas}

Se preparó una serie de soluciones amortiguadoras de $\mathrm{pH}$ 6,5; 6,7; 7,5 y 8,0 disolviendo cantidades adecuadas de $\mathrm{NaH}_{2} \mathrm{PO}_{4} \cdot \mathrm{H}_{2} \mathrm{O}$ y $\mathrm{Na}_{2} \mathrm{HPO}_{4} \cdot 7 \mathrm{H}_{2} \mathrm{O}$ (Merck) para una concentración final de $50 \mathrm{mM}$ en agua desionizada Titripac (Merck). Las soluciones amortiguadoras fueron ajustadas con $\mathrm{NaOH}$ o $\mathrm{HCl}$ utilizando un potenciómetro Thermo Orion 525A+ con un electrodo ROSS Ultra Orion. La respuesta de las membranas PILM y PILM-AB al cambio de $\mathrm{pH}$ fue medida utilizando un Espectrofotómetro UV-Vis UV-mini 1240 Shimadzu.

\section{RESULTADOS Y DISCUSIÓN}

\section{Síntesis de la membrana de líquidos iónicos polimerizados (PILM)}

La membrana de líquidos iónicos polimerizados se preparó mediante la alquilación de 1-vinilimidazol utilizando 1-bromobutano como agente alquilante, obteniendo el líquido iónico bromuro de 3-butil-1-vinilimidazolio [C4vim][Br], el cual se procedió a polimerizar con acrilonitrilo y divinilbenceno, utilizando isobutil éter de benzoína (IEB) como fotoiniciador. El tiempo adecuado para completar la copolimerización es de 10 minutos (figura 1).

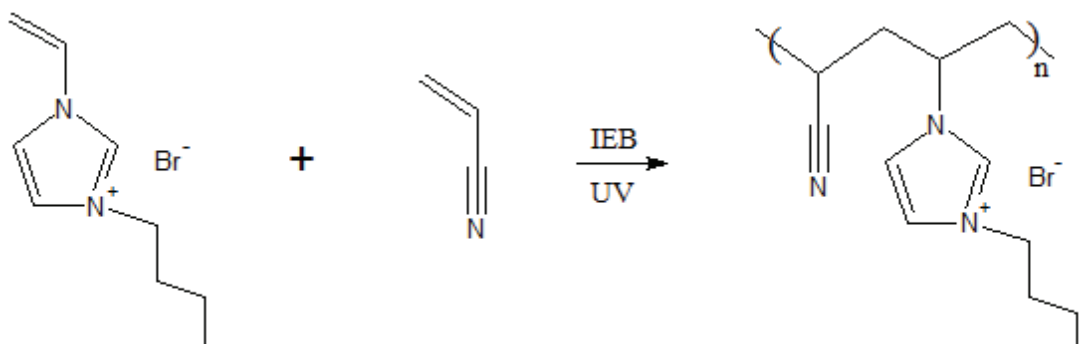

Figura 1. Reacción de copolimerización de bromuro de 3-butil-1-vinilimidazolio y acrilonitrilo. 
La Figura 2 ilustra las características de las membranas a diferentes concentraciones de divinilbenceno como agente reticulante, siendo la cantidad óptima $4 \%(\mathrm{~m} / \mathrm{m})$. Aunque la cantidad relativa de grupos imidazolio es menor al incrementar la concentración de divinilbenceno, el efecto se ve compensado al obtener membranas traslúcidas y con poco hinchamiento.

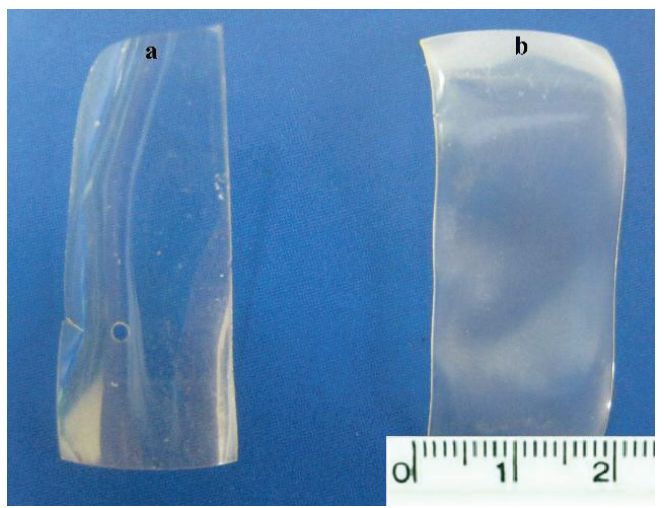

Figura 2. Membranas fotocuradas. (a) Membrana PILM cruda conteniendo $4 \%$ de divinilbenceno y (b) Membrana PILM cruda conteniendo 1\% de divinilbenceno.

\section{Proceso de intercambio iónico}

Las membranas PILM crudas fueron sumergidas en una solución etanólica de amarillo brillante para llevar a cabo el proceso de intercambio iónico. El amarillo brillante posee grupos aniónicos de sulfonato afines al catión imidazolio, los cuales se intercambian por los iones bromuro del IL inicial (figura 3). Las membranas fueron colocadas en agua desionizada y etanol por 6 horas, sin evidenciar lixiviación del indicador.

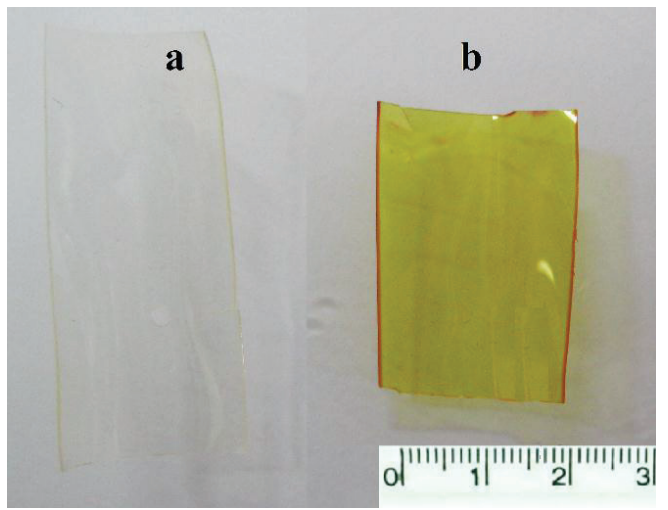

Figura 3. Resultado del proceso de intercambio iónico. (a) Membrana PILM cruda y (b) Membrana cargada con el indicador amarillo brillante PILM-AB. 


\section{Caracterización de PILM y PILM-AB}

Las membranas PILM y PILM-AB fueron caracterizadas mediante FT-IR. Las bandas de absorción de los espectros (figura 4) han sido asignadas en la tabla 1.

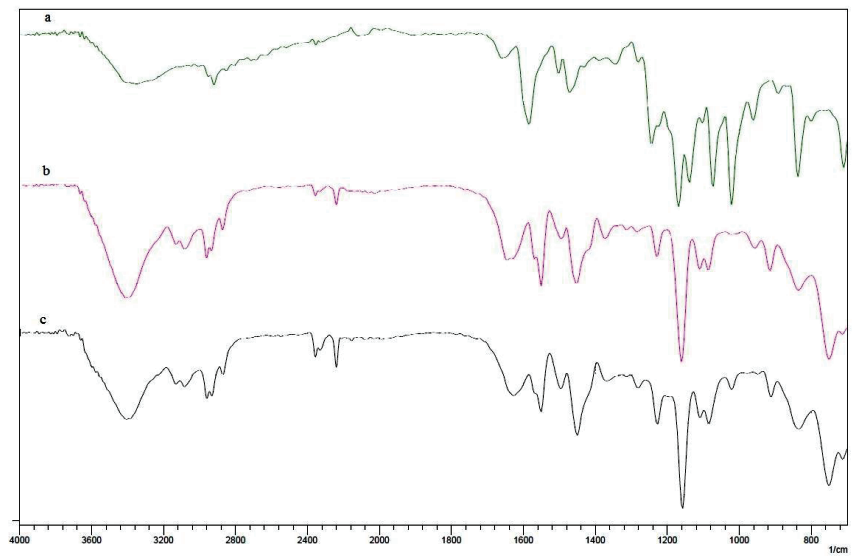

Figura 4. Espectros de Infrarrojo. (a) Indicador Amarillo Brillante, (b) Membrana PILM cruda y (c) Membrana cargada PILM-AB.

Tabla 1. Bandas de Espectroscopía Infrarroja del Indicador Amarillo Brillante, Membrana PILM cruda y Membrana cargada con el indicador PILM-AB $\left(\mathrm{cm}^{-1}\right)$.

\begin{tabular}{|c|c|c|c|c|c|c|}
\hline \multicolumn{2}{|c|}{$\begin{array}{l}\text { Indicador Amarillo B rillante } \\
(\mathrm{AB})\end{array}$} & \multicolumn{2}{|r|}{ PILM } & \multicolumn{2}{|r|}{ PILM-AB } & \multirow{2}{*}{$\begin{array}{c}\text { Ref. } \\
{[14]}\end{array}$} \\
\hline 709 (s) & $\delta$ OH Fuera del plano & & & & & \\
\hline & & $752(s)$ & $\rho$ Catión imidazol & $752(s)$ & $\rho$ Catión imidazol & {$[16]$} \\
\hline 1022 (s) & $v \mathrm{SO}_{3}$ Simétrico & & & $1022(w)$ & $v \mathrm{SO}_{3}$ Simétrico & [14] \\
\hline 1138 (s) & $v \mathrm{~S}=\mathrm{O}$ & & & & & [14] \\
\hline $1168(s)$ & $v$ Anillo asimétrico & $1161(\mathrm{~s})$ & $\checkmark$ Anillo asimétrico & 1157 (s) & $\checkmark$ Anillo asimétrico & {$[15]$} \\
\hline $1242(\mathrm{~m})$ & $v$ C-N Simétrico & $1230(\mathrm{~m})$ & $v$ C-N Simétrico & $1226(\mathrm{~m})$ & $v$ C-N Simétrico & {$[15]$} \\
\hline \multirow[t]{2}{*}{$1284(w)$} & $\rho \mathrm{C}-\mathrm{H}$ Balanceo & $1284(w)$ & $\rho \mathrm{C}-\mathrm{H}$ Balanceo & $1280(w)$ & $\rho \mathrm{C}-\mathrm{H}$ Balanceo & {$[15]$} \\
\hline & & $1450(\mathrm{~m})$ & $\rho \mathrm{CH}_{2}$ Tijereteo & $1450(\mathrm{~m})$ & $\rho \mathrm{CH}_{2}$ Tijereteo & [14] \\
\hline \multirow[t]{2}{*}{$1500(w)$} & $v \mathrm{C}-\mathrm{H}$ & $1496(\mathrm{~m})$ & $v \mathrm{C}-\mathrm{H}$ & $1496(\mathrm{~m})$ & $v \mathrm{C}-\mathrm{H}$ & [16] \\
\hline & & $1550(\mathrm{~m})$ & $v \mathrm{C}=\mathrm{N}$ & $1550(\mathrm{~m})$ & $v \mathrm{C}=\mathrm{N}$ & {$[15]$} \\
\hline \multirow[t]{3}{*}{$1658(w)$} & $v \mathrm{C}=\mathrm{C}$ & 1647 (m) & $v \mathrm{C}=\mathrm{C}$ & $1627(\mathrm{~m})$ & $v \mathrm{C}=\mathrm{C}$ & [14] \\
\hline & & $2241(w)$ & $v \mathrm{C} \equiv \mathrm{N}$ & $2241(w)$ & $v \mathrm{C} \equiv \mathrm{N}$ & {$[13]$} \\
\hline & & $2873(w)$ & $v \mathrm{CH}_{2}$ Asimétrico & $2873(w)$ & $v \mathrm{CH}_{2}$ Asimétrico & {$[15]$} \\
\hline \multirow[t]{5}{*}{$2920(w)$} & $v \mathrm{CH}_{2}$ Asimétrico & & & & & {$[15]$} \\
\hline & & 2935 (m) & $v \mathrm{C}-\mathrm{H}$ & $2931(\mathrm{~m})$ & $v \mathrm{C}-\mathrm{H}$ & {$[15]$} \\
\hline & & $2962(\mathrm{~m})$ & $v \mathrm{C}-\mathrm{H}$ & $2962(\mathrm{~m})$ & $v \mathrm{C}-\mathrm{H}$ & {$[15]$} \\
\hline & & $3082(w)$ & $v \mathrm{C}-\mathrm{H}$ & $3086(w)$ & $v \mathrm{C}-\mathrm{H}$ & {$[15]$} \\
\hline & & $3132(w)$ & $v \mathrm{C}-\mathrm{H}$ & $3132(w)$ & $v \mathrm{C}-\mathrm{H}$ & {$[15]$} \\
\hline \multirow[t]{2}{*}{3352 (m) } & $v-\mathrm{OH}$ en fenoles & & & & & [14] \\
\hline & & 3390 (m) & $v \mathrm{C}-\mathrm{H}$ Anillo vinilimidazol & $3406(\mathrm{~m})$ & $v \mathrm{C}-\mathrm{H}$ Anillo vinilimidazol & {$[17]$} \\
\hline
\end{tabular}

v: Estiramiento, $\rho$ : Flexión, $\delta$ : Deformación, s: Fuerte, m: Medio, w: Débil. 
Los picos que describen al catión imidazolio a $752 \mathrm{~cm}^{-1}$ y al grupo ciano a $2241 \mathrm{~cm}^{-1} \mathrm{se}$ presentan tanto en la membrana cruda PILM y en la membrana cargada PILM-AB. La principal diferencia es la aparición de la señal a $1022 \mathrm{~cm}^{-1}$ perteneciente al estiramiento simétrico del grupo $-\mathrm{SO}_{3}$ en el espectro de la membrana cargada PILM-AB, evidenciando la presencia del indicador en la estructura. También se observa un desplazamiento de la señal correspondiente al estiramiento $\mathrm{C}-\mathrm{H}$ del anillo imidazolio de 3390 a $3406 \mathrm{~cm}^{-1}$, el cual se puede atribuir al efecto del intercambio de aniones bromuro por el indicador amarillo brillante. ${ }^{17}$

\section{Pruebas de respuesta al $\mathrm{pH}$ en soluciones acuosas}

Las membranas PILM-AB fueron colocadas en soluciones amortiguadoras de fosfato de 50 $\mathrm{mM}$ de $\mathrm{pH} 6,5$ y 7,5 por $120 \mathrm{~s}$, pasado este tiempo se realizó el barrido para la obtención de los espectros de absorción (figura 5), los cuales muestran un incremento en la absortividad (efecto hipercrómico) directamente proporcional al incremento del $\mathrm{pH}$, manteniendo la longitud de onda máxima de absorción en 416,0 nm.

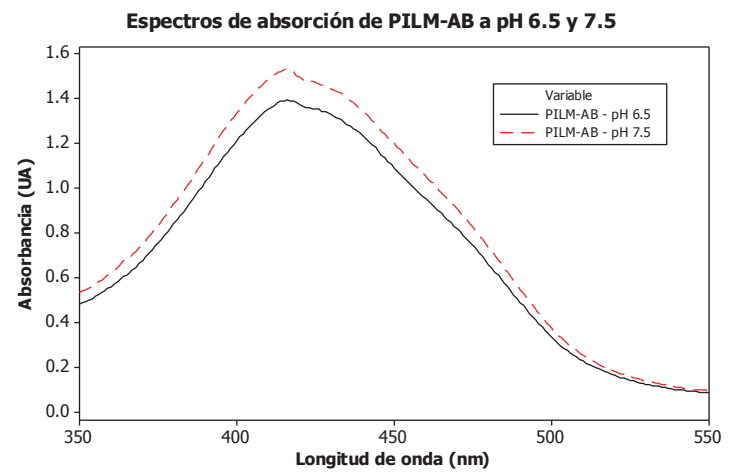

Figura 5. Espectro de absorción de las membranas PILM-AB a pH 6,5 (-) y a pH

$$
7,5(--) \text {. }
$$

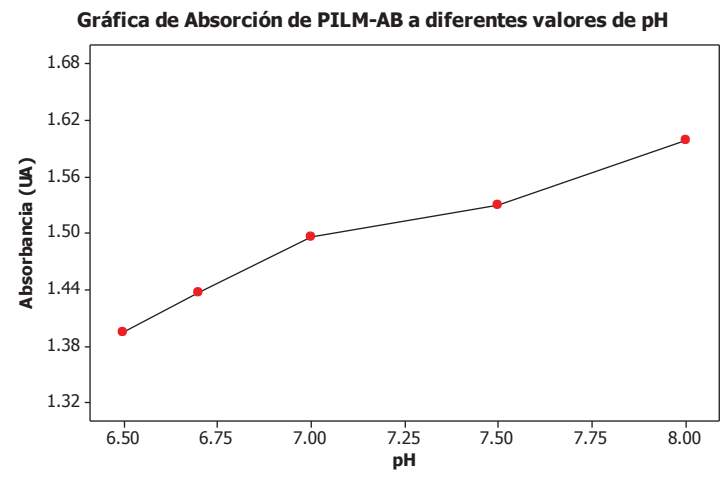

Figura 6. Respuesta de las membranas PILM-AB al incremento del $\mathrm{pH}(\lambda=416,0$ $\mathrm{nm})$. 
Adicionalmente, se llevó a cabo la medición de absorbancia respecto del incremento del $\mathrm{pH}$ de 6,5 a 8,0 a $\lambda=416,0 \mathrm{~nm}$, como se muestra en la figura 6 . Esta relación permite determinar, mediante el uso de un detector, el cambio de color del indicador cargado en las membranas PILM-AB como respuesta al incremento o disminución del $\mathrm{pH}$ en un rango cercano a la neutralidad.

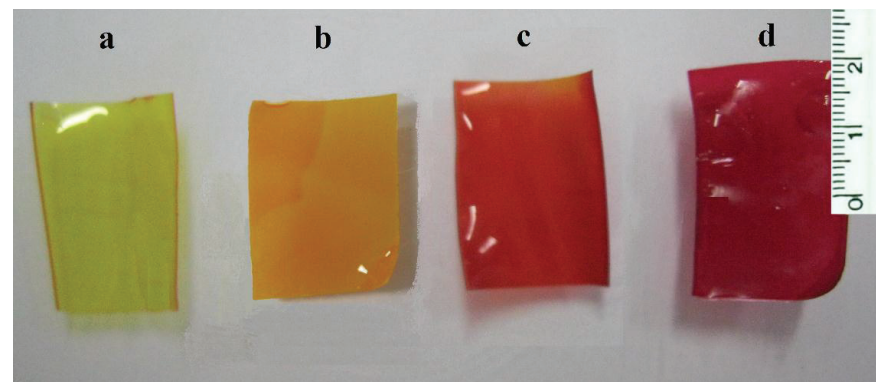

Figura 7. Respuesta de las membranas PILM-AB al incremento de $\mathrm{pH}$. (a) $\mathrm{pH} 6,5$; (b) pH 6,7; (c) $\mathrm{pH} 7,5$ y (d) $\mathrm{pH} 8,0$.

La respuesta de las membranas PILM-AB al cambio de $\mathrm{pH}$ también se pudo determinar de forma visual, como se muestra en la figura 7, obteniendo cambios de color análogos a los de la solución acuosa de amarillo brillante, así como un comportamiento similar en el caso de la reversibilidad, al ser colocadas en las soluciones amortiguadoras en la secuencia inversa, evidenciando que las membranas PILM-AB pueden ser utilizadas repetidas veces, manteniéndose estables y sin lixiviación del indicador.

\section{CONCLUSIONES}

La membrana de líquidos iónicos polimerizados cargada con el indicador amarillo brillante (PILM-AB) puede ser utilizada como medio de detección de cambios de $\mathrm{pH}$ en soluciones acuosas en el rango 6,5 a 8,0; en condiciones cercanas a la neutralidad y sin presentar lixiviación de indicador debido a la alta afinidad del catión imidazolio por los grupos sulfonatos del amarillo brillante. Se han observado cambios significativos en la absortividad de las membranas en respuesta al cambio de $\mathrm{pH}$ a bajas concentraciones $(50 \mathrm{mM})$, lo que es indicativo de una alta sensibilidad de las membranas a estas variaciones. De igual manera, la determinación de la respuesta a la variación del $\mathrm{pH}$ puede efectuarse de manera visual ya que los cambios en la coloración de la membrana cargada son análogos a los cambios del indicador en solución, de manera directa de $\mathrm{pH}$ 6,5 a 8,0 e inversa de $\mathrm{pH}$ 8,0 a 6,5.

\section{AGRADECIMIENTOS}

Agradecimiento a Molident Odontología Integral S.A.C e Innóvate Perú (Convenio 147-FINCyT-FIDECOM-PIMEN 2014) por el apoyo brindado en esta investigación. 


\section{BIBLIOGRAFÍA}

1. Takaya Sato, Takashi Morinaga \& Takeo Ishizuka. Preparation, physicochemical properties and battery applications of a novel poly(ionic liquid). Ed. InTech, 2013. p. 673-695.

2. Jiayin Juan \& Markus Antonietti. Poly(ionic liquids): Polymers expanding classical property profiles. Polymer, 2011; 52: 1469-1482.

3. Fan Liu, Yida Deng, Xiaopeng Han, Wenbin Hu 6 Cheng Zhong. Electrodeposition of metals and alloys from ionic liquids, J. Alloys Compd., 2016; 654: 163-170.

4. Ezzat Rafiee \& Sara Eavani. A new organic-inorganic hybrid ionic liquid polyoxometalate for biodiesel production, J. Mol. Liq., 2014; 199: 96-101.

5. Frank Wendler, Loredana-Nicoleta Todi \& Frank Meister. Thermostability of imidazolium ionic liquids as direct solvents for cellulose, Thermochim. Acta, 2012; 528: 76-84.

6. Anja Balk, Ulrike Holzgrabe \& Lorenz Meinel. Pro et contra ionic liquid drugs Challenges and opportunities for pharmaceutical translation. Eur. J. Pharm. Biopharm., 2015; 94: 291-304.

7. Michael Freemantle. An Introduction to Ionic Liquids. Royal Society of Chemistry, 2010 .

8. Hiroyuki Ohno. Molten salt type polymer electrolytes. Electrochim. Acta, 2001; 46: 1407-1411.

9. Naomi Nishimura \& Hiroyuki Ohno. 15th anniversary of polymerized ionic liquids. Polymer, 2014; 55: 3289-3297.

10. Yuya Egawa, Ryosuke Hayashida \& Jun-ichi Anzai. Multilayered assemblies composed of brilliant yellow and poly(allylamine) for and optical pH sensor. Anal. Sci., 2006; 22: 1117-1119.

11. Cserjési Petra \& Bélafi-Bakó Katalin. Application of ionic liquids in membrane separation processes, Ionic Liquids: Applications and Perspectives. Prof. Alexander Kokorin. Ed. InTech, 2011. p. 561-587. Avaliable from: http://www.intechopen.com/ books/ionic-liquids-applications-and-perspectives/application-of-ionic-liquids-inmembrane-separation-processes

12. J.C. Salamone, S.C. Israel, P. Taylor \& B. Snider. Synthesis and homopolymerization studies of vinylimidazolium salts. Polymer, 1973; 14: 639-644.

13. Jiangna Guo, Lihua Qiu, Zhijun Deng \& Feng Yan, Plastic reusable $\mathrm{pH}$ indicator strips: preparation via anion-exchange of poly(ionic liquids) with anionic dyes. Polym. Chem., 2013; 4: 1309-1312.

14. Joseph B. Lambert. Introduction to Organic Spectroscopy, Nueva York, Macmillan, 1987.

15. Taqiyeddine Moumene, El Habib Belarbi, Boumediene Haddad, Didier Villemin, Ouissam Abbas, Brahim Khelifa \& Serge Bresson. Study of imidazolium dicationic ionic liquids by Raman and FTIR spectroscipies: The effect of the nature of the anion. J. Mol. Struct., 2015; 1083: 179-186.

16. Ji-Lin Wang, Lu-Lu Wang, Rui-Jiang Feng \& Yang Zhang. Synthesis and characterization of novel anion Exchange membranes containing bi-imidazolium-based ionic liquid for alkaline fuel cells. Solid State Ionics, 2015; 278: 144-151. 
17. Xiaofeng Wang, Ligang Xing, Yang Shu, Xuwei Chen \& Jianhua Wang. Novel polymeric ionic liquid microspheres with high exchange capacity for fast extraction of plasmid DNA. Anal. Chim. Acta, 2014; 837: 64-69. 